\title{
Les réformes de politiques publiques en Afrique de l'ouest, entre polity, politics et extraversion
}

\author{
Eau potable et foncier en milieu rural (Bénin, Burkina Faso) \\ Philippe Lavigne Delville \\ Gouvernement et action publique, vol 2018/2 n 2, pp. 53-73 \\ Version auteur
}

\section{Résumé}

L'image de politiques publiques uniformément imposées par les institutions internationales à des Etats africains extravertis et aux faibles capacités institutionnelles ne résiste pas à l'analyse. Si les paradigmes sont issus des prescriptions internationales, les transferts de politiques publiques passent par des processus complexes de réinterprétation et d'adoption sélective. Comparant les réformes des années 2000 sur deux secteurs contrastés et dans deux pays voisins, le Bénin et le Burkina Faso, cet article met au jour des processus contingents, ancrés dans une double histoire politique et institutionnelle, celle des pays et celle des secteurs d'action publique en leur sein. En particulier, l'historicité du secteur, la nature des enjeux politiques qu'il porte, l'existence de controverses au niveau international, et la forme des réseaux de politique publique expliquent le contraste entre les réformes du service de l'eau potable, qui reprennent largement les prescriptions internationales, et celles portant sur le foncier rural, dont la chronologie et les orientations politiques divergent fortement entre les deux pays.

Mots clés : politiques publiques, Afrique, eau, foncier, aide internationale, secteur d'action publique, enjeux politiques

\begin{abstract}
Public policy reform in West Africa: between polity, politics and extraversion. Water supply and rural land tenure (Benin, Burkina Faso)

Public policies in Africa are often seen as being uniformly imposed by international institutions to aid dependant States with few institutional capacities. But such a vision is too simplistic. Policy paradigms follow international prescriptions but policy transfers take place through complex processes of reinterpretation and selective adoption. This paper compares the policy reforms of the 2000s on two contrasted sectors and in two neighboring countries. It highlights contingent processes embedded in the double political and institutional history, of the country and of its different policy sectors. The sectors' historicity, the nature of its specific issues - both in terms of politics and polity - , the existence of controversies at international level, and the configuration of policy networks explain the contrast between the reforms of the drinking water service, which largely incorporate international prescriptions and those concerning rural land tenure, whose chronology and political choices diverge sharply between both countries.
\end{abstract}

Key Words: public policies, Africa, drinking water, land tenure, international aid, public policy sector, politics and polity 


\section{Introduction $^{1}$}

$\mathrm{Du}$ fait des conditionnalités posées par des institutions internationales hégémoniques, du néopatrimonalisme des Etats (Médard, 1991), et de leurs rapports asymétriques avec les institutions d'aide (Whitfield, 2009a) les politiques publiques des Etats africains sont fréquemment vues comme le produit des prescriptions internationales, comme uniformément « imposées par la Banque mondiale ». La dépendance des Etats à l'aide internationale - qui prolonge une extraversion historique (Bayart, 1999) - et leur affaiblissement suite aux ajustements structurels (Coussy, 2006), la capacité de prescription et de transfert de modèles des institutions d'aide (Darbon, 2009), le rôle des experts internationaux dans la définition des politiques (Jampy, 2012), l'ampleur des logiques d'instrumentalisation politique et financière de l'aide (Lavigne Delville, Abdelkader, 2010) et la faiblesse de mouvements sociaux euxmêmes extravertis (Pommerolle, 2010) accréditeraient l'idée que, en dehors de cas spécifiques comme le Bostwana, l'Ethiopie ou le Rwanda (Whitfield, 2009b), les Etats africains et leurs gouvernements ont une faible capacité de porter des projets politiques propres, voire y renoncent dans les logiques de maintien au pouvoir (idem). Le fait même de parler de politique publique dans un tel contexte paraît discutable pour certains auteurs (Darbon, 2004 ; 2015).

De telles analyses mettent incontestablement en avant des caractéristiques structurelles des politiques publiques en Afrique (Enguéléguélé, 2008; Darbon, Crouzel, 2009), dans le contexte de pays «sous régime d'aide» (Lavigne Delville, 2016) où la majorité des investissements publics est financé par l'aide, où l'action publique est largement "multiacteurs et pluri-niveaux» avec un poids important des acteurs internationaux, et où l'imbrication de ces derniers dans les dispositifs publics des Etats s'est encore accrue depuis les années 2000 (Whitfield et Fraser, 2009, p. 19). Pour autant, opposer systématiquement bailleurs de fonds et Etats et analyser leurs rapports de façon unilatérale en termes d'imposition et d'éventuelles résistances sous-estime la diversité et les clivages au sein de ces deux ensembles, et surestime la cohérence des positions au sein des institutions internationales (Fouilleux, 2015) et leur capacité à réellement imposer leurs vues (Thomas, 2004). Très macroscopiques, de telles analyses tendent à généraliser leurs conclusions à l'ensemble des «Etats faibles » sans guère proposer d'analyse comparée de leurs trajectoires spécifiques, ni de la diversité des secteurs d'action publique.

Prenant acte du caractère largement coproduit des politiques publiques en Afrique, je souhaite dans cet article questionner les processus de leur formulation en prenant en compte cette diversité des secteurs et des enjeux. Il s'agit de s'interroger "pas seulement sur l'imposition ou l'auto-imposition des réformes, mais aussi sur leurs parcours complexes par secteur entre policy-making et stratégies politiques »(Darbon, Crouzel, 2009, p. 87).

M'appuyant sur les résultats d'un projet de recherche collectif visant à interroger les conditions de production et d'institutionnalisation de l'action publique en Afrique de l'ouest ${ }^{2}$,

\footnotetext{
1 Je remercie Catherine Baron, Gilles Massardier, Andy Smith, Olivier Provini, les participants au panel du congrès de l'AFSP 2015 organisé par Clothilde Hugon et Olivier Provini, et les relecteurs anonymes de la revue, pour leurs commentaires sur les versions antérieures de cet article.

2 ANR APPI "une action publique éclatée ? Production et institutionnalisation de l'action publique (Bénin, Burkina Faso, Niger) », coordonnée par Catherine Baron (LEREPS/IEP Toujouse). Des études de cas empiriques approfondies, d'orientation socio-anthropologique et d'économie institutionnelle, y ont été menées sur ces deux secteurs et ces deux pays, par une équipe de chercheurs, spécialistes de ces objets, qui les analysaient dans une perspective d'action publique, nouvelle pour eux. Cet article mobilise une partie des résultats de ces travaux, cités au fil du texte et doit beaucoup aux riches débats pendant les différents ateliers qui
} 
j'analyserai les réformes des années 2000 dans deux pays d'Afrique de l'ouest francophone, le Bénin et le Burkina Faso, sur deux secteurs d'action publique, l'eau potable et le foncier en milieu rural. Pays voisins, le Bénin et le Burkina Faso ont connu une histoire politique contrastée dans les dernières décennies, avec pour le Bénin une transition démocratique considérée comme réussie ${ }^{3}$, et pour le Burkina Faso un régime semi-autoritaire chassé par une mobilisation populaire en 2014. Portant sur des ressources naturelles vitales, les deux secteurs de l'eau potable et du foncier posent de façon spécifique la question des tensions et articulations entre régulations coutumières, étatiques et marchandes de la ressource (l'eau, la terre) et du service (l'accès à l'eau potable, la sécurité des droits sur la terre).

La majorité des recherches sur les politiques publiques en Afrique étudient une politique sectorielle dans un seul Etat ${ }^{4}$. Nous proposons ici de dépasser cette perspective monographique en faisant jouer les variables (spécificités du secteur, histoire institutionnelle du secteur, histoire politique et institutionnelle du pays, réseaux d'acteurs) dans ce qu'on peut appeler un comparatisme de proximité.

Nous commencerons par décrire les réformes des années 2000 sur ces deux secteurs, en les situant dans leur histoire. Nous montrerons qu'elles s'inscrivent dans une double dynamique d'intégration des logiques marchandes et d'approfondissement de l'ancrage local de l'Etat, qui prend des formes spécifiques selon les secteurs. Alors que les réformes sur l'eau relèvent de 《principes marchands $\gg^{5}$ globalement partagés et suivent des temporalités voisines dans les deux pays, les temporalités et les trajectoires des réformes foncières sont largement plus contrastées. Nous approfondirons ensuite ce contraste entre les deux secteurs, en montrant qu'il résulte de la nature des enjeux politiques (au double sens de politics et de polity, pris ici au sens de contrat social, de choix de société (Leca, 2012)) du secteur et des tensions que suscitent ou réactivent les projets de réforme.

\section{«Principes marchands » et réformes institutionnelles des années 2000 : les secteurs de l'eau potable et du foncier en milieu rural}

Après les Indépendances, la plupart des pays d'Afrique de l'ouest ont connu une forte instabilité politique et la mise en place de régimes autoritaires, souvent militaires. Les années 1980 et 1990 en Afrique ont été celles de l'ajustement structurel, autour du rétablissement des équilibres macro-économiques, des privatisations, du «désengagement » et du redéploiement de l'Etat (Hibou, 1998). La chute du mur de Berlin légitime des mouvements populaires contre les régimes autoritaires, les bailleurs de fonds soutiennent les transitions au pluripartisme. Pacifiquement ou suite à des révolutions, des régimes fondés sur le libéralisme économique et la compétition électorale se mettent en place au tout début des années 1990

l'ont scandé. Je remercie très sincèrement les collègues du projet de recherche, les discutants et les participants aux différents panels et journées de travail où des versions préliminaires de cet article ont été présentées, et les relecteurs anonymes de la revue pour leurs apports.

${ }^{3} \mathrm{Au}$ sens où le jeu démocratique n’y a pas été remis en cause depuis.

${ }^{4}$ A quelques exceptions comparatives près (Eboko, 2015 ; Provini, Schlimmer, 2016). Pour l'intérêt de la comparaison intersectorielle et inter-pays, au niveau européen, et d'une entrée par le «travail politique » des acteurs, voir Crespy, Ravinet (2014).

${ }^{5}$ Je reprends ici l'expression de Valette et al (2016) pour parler des prescriptions internationales, de préférence à celui de «référentiel marchand» proposé par Baron et Maillefert (2011) dans une perspective d'économie institutionnelle. S'ils entendent bien par là un «ensemble de prescriptions qui donnent du sens à un programme d'action » (Muller, 2010), la notion de référentiel telle qu'elle a été définie par Pierre Muller renvoie à un corps de doctrine en actes et je le réserve au niveau national. On verra aussi qu'il peut exister plusieurs doctrines en compétition, comme dans le cas du foncier. Cf. Fouilleux et Jobert (2017). 
(Buijtenhuijs, Thiriot, 1995). Certains pays consolideront leur ancrage démocratique, quand d'autres connaitront une restauration autoritaire.

Au Bénin, «Etat entrepôt» (Igue, Soule, 1992) dont l'économie est largement structurée autour du port de Cotonou, le coup d'Etat militaire de 1972 a installé au pouvoir le général Mathieu Kérékou, qui adopte en 1974 le principe du marxisme léninisme. La contestation de ce régime, en pleine déroute économique, aboutit en 1990 à la première «conférence nationale » du continent (Banégas, 2003) et à une transition pacifique vers la démocratie. Les premières élections présidentielles de cette nouvelle ère ont lieu en 1991 et voient la victoire de Nicéphore Soglo, ancien administrateur de la Banque mondiale et l'affirmation d'une politique économie libérale. Le Bénin a depuis confirmé son ancrage démocratique et a connu des alternances pacifiques à la tête de l'Etat.

Au Burkina Faso, pays sahélien enclavé, la révolution de 1983 du capitaine Thomas Sankara porte un projet d'autonomie politique face aux grandes puissances, de développement autocentré et de rigueur dans la gestion des affaires publiques. La Haute-Volta devient le Burkina Faso, le «pays des hommes intègres ». Ayant pris le pouvoir en 1987 par un coup d'Etat Blaise Compaoré met en place un régime semi-autoritaire (Hilgers, Mazzocchetti, 2010) couplé à une libéralisation économique. En 1991, il instaure un multipartisme de façade et est élu président de la république. Il sera réélu à plusieurs reprises, jusqu'à sa chute en 2014, du fait de l'opposition populaire à sa volonté de modifier la constitution pour pouvoir briguer un nouveau mandat.

A partir de la fin des années 1990, et plus encore après la chute du mur de Berlin, les Etats africains engagent, sous l'impulsion des bailleurs de fonds, une vaste série de réformes des politiques publiques, de décentralisation administrative et de réorganisations des administrations qui place les bureaucraties et les services publics « en chantier » permanent (Bierschenk, Olivier de Sardan, 2014). Sous couvert de «lutte contre la pauvreté » (Cling, Razafindrakoto, Roubaud, 2002) et de «bonne gouvernance»(Abrahamsen, 2004), elles visent à ancrer institutionnellement la réduction du champ d'intervention de l'Etat. Ces réformes sont censées être élaborées par les Etats, dans une logique «d'ownership » des politiques (Bergamaschi, 2009). Nous analyserons ici les réformes des années 2000 dans les secteurs de l'eau potable et du foncier en milieu rural. Nous les replacerons dans l'histoire du secteur concerné et ses enjeux spécifiques, d'abord de façon générale, au niveau de l'Afrique de l'ouest, avant de détailler leur trajectoire spécifique dans le cas du Bénin et du Burkina Faso.

\begin{tabular}{|c|c|c|c|}
\hline & $\begin{array}{c}\text { Paradigmes } \\
\text { internationaux }\end{array}$ & Bénin & Burkina Faso \\
\hline Régime politique & Ajustements structurels & $\begin{array}{l}\text { 1974-1990: Dictature } \\
\text { marxiste léniniste } \\
\begin{array}{l}\text { 1991- auj : Démocratie } \\
\text { libérale }\end{array}\end{array}$ & $\begin{array}{l}\text { 1983-1987: Révolution } \\
\text { sankariste anti-impérialiste } \\
\text { 1987-1991: Régime } \\
\text { militaire et économie } \\
\text { libérale } \\
\begin{array}{l}\text { 1991-2014 : régime semi- } \\
\text { autoritaire, formellement } \\
\text { démocratique }\end{array}\end{array}$ \\
\hline $\begin{array}{l}\text { Décentralisation } \\
\text { administrative }\end{array}$ & $\begin{array}{l}1990: \quad \text { Décentralisation } \\
\text { administrative }\end{array}$ & $\begin{array}{l}1999 \quad \text { Loi de } \\
\text { décentralisation } \\
\text { 2003: 1ères élections } \\
\text { communales }\end{array}$ & $\begin{array}{l}2004 \text { Code des collectivités } \\
\text { et communalisation } \\
\text { intégrale }\end{array}$ \\
\hline
\end{tabular}




\begin{tabular}{|c|c|c|c|}
\hline & & & $\begin{array}{l}\text { 2006: 1ères élections } \\
\text { communales en zone rurale }\end{array}$ \\
\hline $\begin{array}{l}\text { Eau potable en } \\
\text { milieu rural }\end{array}$ & $\begin{array}{l}\text { Gestion communautaire } \\
\text { 1992 : L'eau comme bien } \\
\text { marchand }\end{array}$ & $\begin{array}{l}\text { 1991: } 1^{\text {ère }} \text { stratégie d'eau } \\
\text { potable, } \\
\text { communautaire }\end{array}$ & $\begin{array}{l}\text { 1998: stratégie sectorielle } \\
\text { eau } \\
2001 \text { loi d'orientation sur } \\
\text { l'eau : mini-réseaux et } \\
\text { délégation de service à } \\
\text { entreprises ou associations } \\
\text { d'usagers }\end{array}$ \\
\hline Foncier rural & $\begin{array}{l}\text { 1975: Privatisation des } \\
\text { terres communes } \\
\begin{array}{l}1990 \text { : Privatisation ou } \\
\text { sécurisation } \\
\text { formalisation des droits } \\
\text { informels }\end{array}\end{array}$ & $\begin{array}{l}\text { Loi de } 1965 \text { sur la } \\
\text { propriété privée }\end{array}$ & $\begin{array}{l}1984 \text { Réorganisation } \\
\text { agraire et foncière (RAF) : } \\
\text { nationalisation des terres } \\
\begin{array}{l}\text { Divers projets } \\
\text { expérimentaux }\end{array} \\
\begin{array}{l}2009 \text { : Politique nationale } \\
\text { de sécurisation foncière : } \\
\text { sécurisation et gestion } \\
\text { communale }\end{array}\end{array}$ \\
\hline
\end{tabular}

Tableau 1. Présentation synthétique des cas

Paiement au volume et délégation au privé de la gestion des équipements dans l'eau potable

L'histoire des politiques dans le secteur de l'eau potable en milieu rural peut se lire en trois temps (Baron, Maillefert, 2011 ; Diop, Hamath Dia, 2011). Dans un premier temps, l'Etat (colonial puis postcolonial) prend en charge la construction de puits cimentés dits « modernes » ou de forages équipés de pompes à motricité humaine, et en assure la gestion et la maintenance. L'eau potable est alors conçue comme un bien public, dont l'Etat doit assurer la fourniture. En 1980, la Décennie internationale de l'eau potable et de l'assainissement (DIEPA), lancée par l'ONU, pousse à étendre l'accès à l'eau potable, principalement sous forme de forages manuels. Dans un second temps, avec les politiques d'ajustement structurel, la gestion au quotidien des équipements et leur maintenance sont transférées aux populations sous le label de "gestion communautaire ». Du fait des performances très variables des comités de gestion et des carences de l'environnement institutionnel, cette gestion communautaire rencontre des succès mitigés (Olivier de Sardan, Dagobi, 2000; Bonnassieux, Gangneron, 2011), elle aboutit fréquemment à une privatisation de fait du contrôle de l'équipement par une faction ou un commerçant. L'Etat et les projets de développement doivent régulièrement réhabiliter des équipements en panne, dont le statut juridique est peu clair.

Le principe de l'eau comme bien marchand se diffuse au niveau international à partir de la Conférence internationale sur l'eau et l'environnement de Dublin, en 1992, et est rapidement promu dans les pays africains. C'est le troisième temps : l'eau potable est désormais conçue comme un bien marchand, elle doit être vendue au volume pour assurer la rentabilité des équipements, laquelle est censée garantir la pérennité du service. Ce principe marchand est appliqué avec peu de succès aux forages manuels existants. Il trouve sa concrétisation avec le modèle des mini-adductions d'eau potable, diffusé au cours des années 1990 : destiné aux 
gros villages ou aux petits villes, qui étaient auparavant desservis par un ensemble hétérogène de puits privés et de forages à main, ce modèle repose sur un forage motorisé, un château d'eau, un petit réseau de bornes fontaines où la vente de l'eau est assurée par des fontainières, rémunérées ou indemnisées à partir des recettes. Les comités de gestion sont recomposés en associations d'usagers, aux règles internes plus structurées (Baron, Bonnassieux, 2013). La mise en place des communes (Bénin, 2003; Burkina Faso, 2006) induit une nouvelle réorganisation institutionnelle : les communes reçoivent la responsabilité de l'extension et du bon fonctionnement des équipements d'eau potable.

Malgré de réelles réussites, la délégation de responsabilité à des instances «communautaires » peu définies n'a globalement pas permis d'assurer une maintenance durable des équipements. La gestion communautaire est à son tour mise en cause au cours des années 2000. En cohérence avec le principe néolibéral de séparation de la propriété des infrastructures et de leur gestion, la propriété des équipements et la responsabilité du service de l'eau sont données aux communes, la gestion est déléguée à des opérateurs privés.

Au Burkina Faso, le secteur de l'eau est réorganisé par une série de textes au tournant des années 2000 : Document stratégique en 1998, décret réformant le système de gestion des infrastructures hydrauliques d'alimentation en eau potable en milieux rural et semi urbain en novembre 2000, le tout englobé début 2001 dans la «Loi d'orientation relative à la gestion de l'eau ». Le principe de la délégation de la gestion des réseaux à un opérateur privé est posé dès le départ, mais les débats sont vifs sur la nature des organisations pouvant en bénéficier : pour renforcer les associations villageoises de gestion de l'eau, un ancien directeur régional de l'hydraulique a promu dans les années 1990 dans la région de Bobo Dioulasso une fédération appuyé par une organisation professionnelle, l'ADAE (Association pour le développement des adductions d'eau dans la région de Bobo-Dioulasso). Du fait de ce précédent, et de la légitimité de son fondateur, l'ADAE a réussi à obtenir une interprétation souple du principe de délégation, qui peut se faire au profit d'associations locales (Baron, Bonnassieux, 2013).

Au Bénin (Bonnassieux, Gangneron, 2011; Valette, Gangneron, Bonnassieux, 2016), la première stratégie d'approvisionnement en eau potable date de 1992. Traduisant le second temps des politiques de l'eau, elle énonce un principe de réponse à la demande, de participation financière, et délègue la gestion des points d'eau aux communautés locales, sous forme de «Comités de gestion des points d'eau » ou «d'Associations d'usagers de l'eau » pour les réseaux. La décentralisation, mise en place en 2003 sur un dispositif juridique adopté en 1999, donne aux communes la responsabilité du service de l'eau et prévoit la possibilité de délégation du service. La nouvelle réforme du secteur, au cours des années 2000, intègre ainsi les communes dans le dispositif institutionnel, mais la tension entre logique territoriale et logique sectorielle demeure vive, les agents du ministère de l'eau étant très réticents au transfert. La politique de l'eau a été révisée en 2004, elle constitue la base de la «Stratégie nationale d'approvisionnement en eau potable et assainissement en milieu rural $\gg$ (20052015) puis de la loi de 2008 sur la gestion de l'eau, qui oblige les communes à déléguer la gestion des réseaux à un opérateur privé. La version de 2005 de la stratégie prévoyait en réalité des modalités de délégation aux associations d'usagers (Bonnassieux, Gangneron, 2011), qui ont été en pratique écartées dès 2007 par les cadres du ministère (Valette et al, op. cit.).

Bénin et Burkina Faso connaissent, depuis les années 1990, une diffusion progressive de principes marchands. Promus à l'échelle internationale, diffusé par les institutions d'aide, ces principes reposent sur la vente de l'eau au volume. Mais ils ne peuvent en pratique véritablement s'imposer (et s'affirmer comme référentiel du secteur dans les deux pays) que dans la décennie 2000, lorsque les dispositifs techniques sont adéquats (les mini réseaux) et le 
cadre institutionnel global consolidé (des communes ayant légalement responsabilité du service et aptes à en déléguer la gestion).

Les années 2000 correspondent alors à une phase d'achèvement institutionnel de la construction du secteur, dans un contexte global néolibéral, fondé sur une conception d'un Etat minimal, qui promeut le marché, le recours aux acteurs privés dans l'action publique, la délégation et la sous-traitance, et une participation formelle des usagers. Les modalités concrètes des réformes prennent cependant des formes différentes dans les deux pays, en lien avec deux facteurs : d'abord la temporalité respective de la réforme du secteur de l'eau et de la décentralisation, le transfert aux communes de la responsabilité du service de l'eau étant plus difficile à intégrer dans la politique de l'eau lorsque la décentralisation lui est postérieure, et ensuite des choix différents quant aux formes de délégation. Ces réformes relèvent dans les deux cas de la conjonction entre des bailleurs de fonds, des hauts cadres du secteur et des experts, souvent issus de l'administration, qui mettent en avant la nécessaire professionnalisation du service, disqualifient la gestion communautaire et cherchent à promouvoir de nouveaux arrangements institutionnels, tout en s'appuyant en partie sur des expériences préalables dans le pays.

La traduction pratique de ces réformes sur le terrain est variable (Baron, Tidjani Alou, 2011). L'exigence accrue de rentabilité et les fréquentes augmentations de prix accentuent les risques d'exclusion des pauvres de l'accès au service, et mettent en cause les compromis entre logique de marché et logiques sociales qui avaient été négociés autour des pompes à motricité humaine et des comités de gestion: la souplesse pratique dans l'exigence de paiement permettait de réunir des fonds tout en exemptant en pratique les pauvres et les proches des fontainières. Au Bénin, les Associations d'usagers ont tenté de résister à ces réformes qui les excluaient du service et, au-delà des postes politiques et des rentes qu'ils permettaient, remettent en cause la possibilité de réinvestir une partie des bénéfices de la gestion communautaire dans des investissements sociaux locaux (Bonnassieux, Gangneron, 2011).

\section{Entre reconnaissance et privatisation : formalisation et administration des droits fonciers coutumiers}

L'histoire du secteur du foncier rural en Afrique de l'ouest peut se lire en deux temps. Aux Indépendances, les Etats ${ }^{6}$ ont conservé le dualisme légal colonial, qui organise une séparation entre les terres privées, objets de titres fonciers délivrés suite à la procédure d'immatriculation foncière, et le domaine privé de l'Etat, qui intègre toutes les terres non immatriculées, c'est-àdire la grande majorité du territoire, du fait du coût et de la complexité de l'immatriculation. Ils ont mis de côté les tentatives du colonialisme tardif pour ouvrir une possibilité de formalisation des droits fonciers coutumiers (décrets AOF de 1955 et 1956). Jusqu'aux réformes récentes (et même jusqu'à aujourd'hui en pratique, à cause de leur faible déploiement sur le terrain), la quasi-totalité des terres rurales dans ces pays relève de régulations coutumières ou néo-coutumières.

Le deuxième temps s'ouvre à la fin des années 1980, lorsque la question foncière rurale, longtemps considérée comme secondaire, apparaît à l'agenda international, à la croisée des ajustements structurels qui prônent le marché foncier comme condition du développement économique, de la contestation du monopole foncier de l'Etat et des abus de pouvoir auxquels il a donné lieu, et d'une montée des conflits fonciers en milieu rural. L'absence de reconnaissance légale des droits fonciers des exploitants agricoles est perçue comme une des sources de l'insécurité foncière et un frein au développement agricole, et la formalisation de

\footnotetext{
${ }^{6}$ A l'exception du Sénégal, qui a placé les terres rurales dans le Domaine national et en a confié la gestion aux Communautés rurales, circonscriptions administratives de base (Le Roy, 1985).
} 
ces droits devient le mot d'ordre. Deux grandes doctrines s'affrontent cependant (Bruce, Freudenberger, 1992) : celle de la privatisation, qui prône la suppression rapide des droits coutumiers jugés inefficaces et de la privatisation des droits sur la terre au nom de l'efficacité économique, et celle de la sécurisation foncière, qui met en avant l'efficacité et la capacité d'adaptation des droits fonciers locaux/coutumiers, et pour laquelle la priorité est de construire un cadre institutionnel sécurisant ces droits tout en leur permettant d'évoluer au rythme des changements économiques et sociaux.

Les pressions internationales à la privatisation des terres n'ont guère d'écho au niveau des Etats à cette époque, d'autant qu'elle est contestée au sein même des organisations internationales. La question de la gestion des conflits, de la sécurisation des droits fonciers locaux, est à l'ordre du jour en Afrique, poussée à la fois par des cadres nationaux travaillant en milieu rural et par des acteurs internationaux, mais le foncier est considéré comme politiquement trop sensible, et les élites préfèrent maintenir un dualisme légal qui favorisent leur accumulation foncière. Des expériences de reconnaissance et de sécurisation des droits fonciers coutumiers sont menées dans des projets de développement expérimentaux. Les débats sur les politiques foncières rurales connaissent une histoire complexe, avec de multiples tentatives de mise sur agenda et peu d'aboutissements. C'est au cours des années 2000 que quelques pays adoptent des réformes, à la fois légales et institutionnelles, d'orientations variées, et dont l'opérationnalisation dans l'espace rural est très progressive. Vingt cinq ans après la mise sur agenda du foncier à l'échelle internationale, la formalisation légale élargie des droits sur la terre n'est pas encore concrétisée.

Au Bénin, la question de la sécurisation foncière rurale émerge dans le cadre d'un projet de développement et de gestion des ressources naturelles, préparé par la Banque mondiale, la Caisse centrale de coopération économique (aujourd'hui Agence française de développement, AFD) et la GTZ (agence allemande de coopération technique, aujourd'hui GIZ) à la fin des années 1980, dans les derniers moments de la dictature de Mathieu Kérékou. L'expérimentation de Plans fonciers ruraux, démarche de cartographie des droits fonciers coutumiers, individuels ou collectifs est dès cette époque conçue comme devant permettre d'alimenter une future réforme légale octroyant des droits juridiquement reconnus aux paysans (Lavigne Delville, 2010).

Le régime révolutionnaire n'avait pas nationalisé les terres, ni modifié la loi de 1965 sur la propriété. Dans les années 1990, dans le contexte d'un Bénin démocratique à l'économie libérale, l'inadaptation de ce cadre légal est patente et différents ministères (urbanisme, agriculture, entreprises) initient sans succès des projets de réforme, avec des orientations différentes. En 1999, conformément à ses engagements auprès des bailleurs de fonds, le gouvernement met en place un comité d'experts béninois pour préparer un projet de loi définissant un nouveau régime foncier rural. Sur un sujet aussi sensible, très fortement politisé, il refuse de recourir à de l'expertise internationale pour conserver la maitrise de ses choix. Après de longues consultations et négociations internes, ce comité rédige un avantprojet de loi qui propose une véritable révolution juridique : le texte propose d'intégrer les «terres objets de droits établis et acquis selon la coutume et plus largement les normes et pratiques locales» au sein des «terres privées». Rompant avec la présomption de domanialité sur les terres non immatriculées, créant un nouveau statut juridique de la propriété, qui n'est plus garanti par l'Etat mais peut être contestable devant les tribunaux, mettant en place un dispositif d'administration foncière de proximité intégré aux nouvelles communes, ce texte constitue une «politique instituante » (Jobert, 1998) qui redéfinit la façon de penser et organiser un secteur.

Prêt dès 2001, ce texte sera finalement voté en 2007 dans un contexte paradoxal. A partir de 2004, en effet, le MCA-Bénin (équipe béninoise mise en place par le gouvernement et 
financée par le Millenium Challenge Corporation, nouvelle agence d'aide américaine créée en 2004) et le Ministère de l'urbanisme lancent une initiative de réforme foncière, couvrant le rural comme l'urbain. Partie intégrante d'un programme de 5 ans visant à stimuler la croissance économique par l'investissement, le volet foncier du MCA (2006-2011) vise à «faire de la terre un actif monnayable ». Pour ses promoteurs, en cohérence avec la doctrine de la privatisation, la solution aux nombreuses carences de la régulation foncière (informalité, corruption, conflits, etc.) consiste à démultiplier la délivrance de titres de propriété privée en simplifiant les procédures et en réduisant les coûts, et à réformer l'administration foncière, centralisée, jugée corrompue et inefficace, en mettant en place une agence nationale dotée de bureaux déconcentrés.

Cette initiative met en cause les bases de la réforme foncière rurale en cours d'institutionnalisation et marginalise le Ministère de l'agriculture. Elle est fortement contestée par les spécialistes du foncier rural, les bailleurs de fonds du secteur, un collectif d'organisations de la société civile (Lavigne Delville, Saïah, 2016). Elle aboutit malgré tout en 2013 au vote d'un «Code domanial et foncier », qui abroge les textes antérieurs, y compris la récente loi foncière rurale de 2007 : si les Plans fonciers ruraux sont intégrés dans le Code, c'est en tant que moyens pour délivrer des titres de propriété privée individuelle, gérés par la future agence de gestion foncière, et non plus la base de certificats fonciers individuels ou collectifs, gérés au niveau des communes.

Après plus d'un an de féroces luttes interinstitutionnelles entre ministère de l'urbanisme, qui a porté le dossier depuis une dizaine d'années, et ministère des finances, qui avait historiquement la tutelle de l'administration foncière, ce dernier gagne en 2014 la tutelle de l'Agence. La mise en œuvre se met très progressivement en place, avec le soutien financier des Pays-Bas, qui entrent à ce moment-là comme bailleurs de fond du secteur, et n'est pas encore effective sur le terrain, faute que les instruments cadastraux soient disponibles. Pendant ce temps, les 300 Plans fonciers ruraux, qui avaient été réalisés «à marche forcée » entre 2007 et 2011 se délitent : le cadre institutionnel prévu par la loi de 2007 a été aboli par le nouveau Code mais l'agence n'est pas encore en place; marginalisées dans le Code, les communes se désinvestissent.

$\mathrm{Au}$ Burkina Faso, la politique foncière s'incarne dans la Réorganisation Agraire et Foncière (RAF), projet central du régime de Thomas Sankara, promulguée symboliquement le jour du premier anniversaire de la révolution. Dans une optique largement étatiste, la RAF avait pour objectif de marginaliser les pouvoirs coutumiers et de promouvoir une gestion " rationnelle » des terres, fondée sur une nationalisation des terres et l'interdiction du marché foncier. La RAF est amendée en 1991 puis 1996 dans un contexte de libéralisation économique, sans vraiment affronter les problèmes qu'elle pose dans le milieu rural, où les régulations coutumières sont dominantes. Des projets de développement, financés par différents bailleurs de fonds (France, Allemagne, Pays-Bas, Banque mondiale) ont, dans les années 1990, tenté de trouver des réponses aux conflits fonciers, sans reprise politique de leurs démarches. Au milieu des années 2000, "vingt ans après [la promulgation de la RAF], l'impression de piétinement, de gaspillage des initiatives domine » (Tallet, 2009, p. 141-142).

En 2004 pourtant, le ministre de l'Agriculture, numéro deux du régime à cette époque, donne son accord pour un processus participatif d'élaboration d'une «Politique nationale de sécurisation foncière rurale », qui se déroulera entre 2005 et 2007, aboutissant à la «Loi portant régime foncier rural » en 2009. Le compromis fondateur est que la politique foncière rurale doit permettre de sécuriser les acteurs ruraux, paysans comme entrepreneurs. La politique nationale de sécurisation foncière rurale crée des mécanismes de reconnaissance des droits fonciers paysans à travers les «attestations de possession foncière rurale », met en place des services fonciers au niveau des communes rurales (Jacob, 2013; Hochet, Jacob, 
2014). La mise en œuvre de la politique a démarré en 2009 dans le cadre de projets financés par l'AFD et le MCC (via le MCA-Burkina Faso) : des services fonciers communaux ont été mis en place dans une première série de communes, la délivrance des attestations de possession foncière a débuté. Elle a connu une phase d'incertitude avec la fin des premiers financements et la chute de Blaise Compaoré, entérinant un déploiement partiel et hétérogène dans le pays.

Ces deux processus de réforme foncière s'inscrivent dans un contexte de contestation du monopole foncier de l'Etat sur les terres non immatriculées, de développement non régulé du marché foncier, et de mise en avant de l'enjeu de la reconnaissance légale des droits locaux. Mais leur chronologie et leurs orientations politiques sont très différentes. Le Bénin, qui avait démarré plus tôt les expérimentations de terrain, semblait au début des années 2000 engagé dans une politique très novatrice de sécurisation foncière rurale, mais l'offre de financement du MCC a permis aux promoteurs de la privatisation de reprendre l'initiative et finalement de l'emporter. Au Burkina Faso, la question du foncier rural a longtemps été politiquement bloquée, la mise sur agenda été plus tardive, mais une fois l'impulsion donnée, tout est allé très vite. La spécificité du foncier rural a été reconnue par les différents ministères sectoriels concernés. La « Politique nationale de sécurisation foncière rurale » va même plus loin dans ce sens que la loi foncière rurale de 2007 au Bénin, car elle institutionnalise la négociation locale des règles de gestion des terres et des ressources naturelles au sein de chartes foncières, et rend facultative le recours aux attestations de possession foncière.

\section{Politics, polity et trajectoires des réformes}

A des degrés variables, les réformes des années 2000 s'inscrivent dans le référentiel global néo-libéral mis en place pendant les ajustements structurels. Sur le secteur de l'eau potable, les options sont la valorisation du marché, la réduction du périmètre d'intervention directe de l'Etat, le principe de décentralisation et de délégation au privé de la mise en œuvre du service et de la gestion des équipements. Le référentiel marchand n'est guère contesté au sein des réseaux de politique publique, les nuances se jouent dans la gamme des formes de délégation. Sur le secteur du foncier, la question du marché foncier est explicitement ou implicitement au cœur des débats sur les réformes: le favoriser est un objectif explicite des politiques de privatisation (Manji, 2006); les politiques de sécurisation ne promeuvent pas les marchés fonciers de façon volontariste mais, en offrant un cadre juridique aux terres objets de droits coutumiers et en mettant en place des procédures pour formaliser les transactions foncières marchandes, elles entérinent et favorisent en pratique leur développement. De ce point de vue, on peut considérer que ces réformes relèvent d'un ajustement global/sectoriel (Muller, 2010) au référentiel néo-libéral post-ajustement, au sens où les référentiels qui gouvernaient auparavant les secteurs (la gestion communautaire pour l'eau potable, l'immatriculation foncière comme seule forme d'accès au droit pour le foncier et l'intégration des droits fonciers informels dans le domaine privé de l'Etat pour le foncier) sont désormais disqualifiés et où les réformes visent à mettre l'organisation du secteur en conformité avec les principes marchands (et avec la décentralisation administrative, les instances communales étant responsables du service de l'eau, et aussi des nouveaux dispositifs de gestion foncière dans les politiques de sécurisation foncière).

Pour autant, il ne s'agit pas seulement d'institutionnaliser un retrait de l'Etat. Dans les deux secteurs, l'Etat organise l'extension du service dans l'espace rural (service de l'eau et administration foncière), différencie ses politiques et ses instruments, met en place de nouveaux dispositifs institutionnels à l'échelle locale, impose ses normes et sa façon d'organiser le secteur. Bref, il se redéploie autant qu'il se désengage (Hibou, 1998). Les 
réformes des années 2000 s'inscrivent en fait dans un triple processus de différenciation progressive du cadre légal et institutionnel (Jacob, 2013 ; Hochet et Jacob, 2014), d'extension de la présence de l'Etat sur le territoire (idem) et enfin d'ajustement global/sectoriel des politiques. Le premier et le second poursuivent des processus historiques d'affirmation de l'Etat, démarré dès l'époque coloniale; le troisième prend acte de processus de marchandisation des ressources; malgré l'ajustement structurel et les pressions au désengagement de l'intervention directe, il recompose les dynamiques de long terme plus qu'il ne les annule.

Bien plus, cette mise en conformité est loin d'être mécanique. Les prescriptions internationales diffèrent selon les secteurs, elles sont réinterprétées par les acteurs nationaux des politiques publiques, nationaux comme internationaux, en fonction de l'économie politique du secteur, mais aussi de visions politiques. Un secteur est « une forme d'institutionnalisation de la division du travail gouvernemental », « constitué par un ensemble de problèmes associés de manière plus ou moins institutionnalisée à certaines populations », et constitue un espace «d'élaboration de normes et valeurs spécifiques » (Muller, 2010, p. 405 et 407). Le contraste entre les réformes sur l'eau potable et le foncier, d'une part, entre les options prises par le Burkina Faso et le Bénin sur le foncier, d'autre part, éclaire la façon dont les réformes, leurs temporalités, leurs résultats sont enchâssés dans les enjeux politiques (en termes de politics comme de polity) du secteur et du pays.

\section{Eau potable vs foncier: enjeux politiques des secteurs et rapport aux prescriptions internationales}

L'eau potable est un enjeu politique au sens où les questions de la ressource et de sa propriété, de son coût de production et de distribution et de la façon de l'assumer, des inégalités d'accès et de coût, comportent des enjeux en termes d'inclusion ou d'exclusion, de centralisation ou de décentralisation. Mais ce n'est pas un enjeu fort en termes d'économie politique à l'échelle nationale. Les controverses qui traversent les réformes renvoient au contrôle du secteur, entre administration sectorielle et décentralisation, autour de la question de la maîtrise des financements internationaux et de la mainmise sur les appels d'offres pour la réalisation des adductions d'eau. A l'échelle locale, les luttes portent sur la rente liée à la gestion du service et sur le pouvoir de choisir les délégataires du service. Le débat entre gestion privée et associative recouvre en outre une tension sur la conception du service: les associations d'usagers utilisent parfois leurs bénéfices pour des investissements collectifs villageois, elles prennent parfois à leur charge des extensions de réseaux ou peuvent décider de ne pas faire payer certains acteurs ${ }^{7}$.

En effet, le principe de régulation marchande est en tensions avec les normes sociales locales. En rendant possible des exclusions par la pauvreté, il met en question la norme coutumière selon laquelle on ne refuse pas l'eau de boisson à qui en a besoin. Il s'oppose au principe d'un traitement privilégié des parents et amis. Le principe du paiement au volume n'est guère mis en cause par les usagers, à la fois parce qu'il a déjà une histoire, et parce que, dans les normes sociales locales, il est normal de payer pour un travail et que, contrairement aux pompes à main, l'eau des mini-réseaux implique des coûts de pompage. Mais les usagers peuvent par contre contester son coût. S'ils le jugent trop élevé pour eux, ils peuvent retourner aux puits ou aux pompes manuelles, ou tenter de négocier avec les fontainières des arrangements locaux avec la norme : avec l'extension de la contrainte de rentabilité et la mise en place de compteurs aux bornes fontaines, ce sont désormais elles fontainières qui assument la

\footnotetext{
${ }^{7}$ Cf. Diop et Hamath Dia (2011) pour le Sénégal.
} 
contradiction entre normes locales et principe marchand, et voient le manque à gagner lié à ces exemptions déduites de leur petit revenu.

Mais ce problème n'est pas posé dans les débats sur la politique de l'eau. Les organisations non gouvernementales ont des positionnements variés, mais partagent globalement ce référentiel marchand, par adhésion ou faute d'alternative crédible. L'absence de controverses fortes sur ce référentiel, le peu d'enjeux d'économie politique, contribuent à expliquer que les deux pays étudiés aient connu des réformes assez similaires, et que les évolutions des doctrines internationales se soient traduites assez rapidement en réformes.

Les enjeux sont très différents sur le secteur du foncier. A la fois enjeu de richesse, pouvoir et sens (Shipton, Goheen, 1992), le foncier est au cœur de l'économie politique et des processus de formation de l'Etat (Boone, 2013). Les élites nationales ont largement utilisé à leur profit une législation complexe et accaparé des parts significatives du «domaine national », au détriment des paysans et des éleveurs. La prévalence de l'informalité autorise des stratégies de «gestion de la confusion » (Mathieu, 1996) qui servent les intérêts des élites économiques et politiques, et ceux des agents de l'administration foncière, réputée être un haut lieu de corruption.

Au-delà des dynamiques de spéculation foncière et des luttes d'intérêts corporatistes, le foncier pose des questions de choix de société, plus fondamentaux que l'eau potable. Dans les normes coutumières, l'accès à la terre est médiatisé par la position dans les systèmes d'inégalités et de domination, tant entre autochtones et migrants, qu'au sein des groupes familiaux. Dans des contextes où - au-delà de la grande diversité des configurations locales l'essentiel des régulations foncières en milieu rural relève de normes coutumières plus ou moins métissées de principes étatiques et où des transactions marchandes non régulées se développent, la controverse entre sécurisation foncière et privatisation dépasse largement la question des politiques agricoles. Elle recouvre des débats fondamentaux - mais pas toujours explicites - sur les rapports entre individus, collectifs sociaux (familiaux et ethniques) et Etat, sur l'équilibre ou les tensions entre citoyenneté locale et citoyenneté nationale, sur les rapports entre pouvoirs coutumiers et Etat.

Enjeux d'économie politique et enjeux de société se croisent donc pour faire des politiques foncières un objet particulièrement controversé. Le foncier est de plus un objet à la croisée de multiples champs professionnels et d'organisations. Il est moins le monopole d'un corps professionnel, d'un ministère. Ces confrontations d'idées et d'intérêts s'expriment d'autant plus fortement que les institutions internationales sont elles aussi traversées par un clivage entre sécurisation et privatisation, entre défense de l'agriculture familiale et promotion de l'agro-business. Pour toutes ces raisons, les processus nationaux de réformes sont lents, complexes, moins standardisés. Des jeux d'intérêts contraires peuvent bloquent les tentatives de réformes, ou entraîner un basculement d'un cadrage à un autre.

Des choix divergents dans les réformes foncières au Bénin et au Burkina Faso : le rôle des réseaux de politique publique

Au Burkina Faso comme au Bénin, les années 2000 ont vu une accélération des achats de terres par les élites urbaines, et la promotion de l'agro-business comme base des politiques agricoles. L'imaginaire de la propriété privée, la visibilité des achats de terres par les urbains en périphéries urbaines, sont plus visibles au Bénin. Les marchés fonciers y sont plus institutionnalisés, les communes enregistrant les conventions de vente. Mais c'est l'histoire des réformes et les réseaux de politiques publiques qui les portent ou s'y affrontent, plus que des enjeux d'économie politique pas fondamentalement différents, qui explique la divergence des trajectoires. 
Au Bénin, deux réseaux s'opposent, qui traversent tous les deux les frontières entre national et international (Lavigne Delville, 2010) : la doctrine de la sécurisation foncière est porté par un réseau réunissant des bailleurs de fonds européens, un noyau de spécialistes travaillant dans les projets pilote, des cadres du ministère de l'agriculture et des experts défenseurs de l'agriculture familiale, ainsi que d'autres acteurs qui connaissent le milieu rural et ne pensent pas que la privatisation des terres soit possible ou souhaitable. Il est rejoint tardivement par un syndicat paysan et une coalition d'ONG qui tentent de se battre contre le projet de Code domanial et foncier (Lavigne Delville, Saïah, 2016). Le caractère insuffisamment formalisé de son corps de croyance, la faiblesse de ses liens dans l'appareil d'Etat, en font un "réseau sur enjeu » qui ne parvient pas à se structurer en véritable " communauté de politique publique » (Thatcher, 2004). Le second réseau se structure autour du Ministère de l'urbanisme et de l'équipe du MCA, il est est critique des pratiques de l'administration foncière mais défend une conception classique, juridique et topographique, du secteur. Cohérente avec l'orientation clairement libérale du régime politique, l'offre de financement du MCC leur donne l'opportunité de promouvoir une réforme ambitieuse, où ils sont rejoints par les professionnels du secteur (géomètres, notaires) qui y trouvent des intérêts corporatistes. Plus structuré, ayant l'appui des professionnels, porteur d'un discours simple sur les bienfaits de la propriété privée, ce réseau peut être considéré comme une "communauté de politique publique » au sens fort du terme, "composée de fonctionnaires des ministères et de groupes d'intérêt, qui font les politiques, nouant des relations d'échanges et se construisant une identité commune» (idem : 385). Mieux positionné dans le gouvernement, doté de soutiens financiers importants, il l'emportera finalement.

Au Burkina Faso, les configurations sont radicalement différentes (Lavigne Delville, Thieba, 2015) Les différents projets expérimentant des stratégies de sécurisation foncière rurale ont permis à un noyau d'experts engagés de se construire une vision. Ils ont fondé une association d'échanges, où des acteurs variés, cadres de l'administration, consultants, chercheurs, se sont progressivement constitué une analyse partagée. Alors même que le Ministre de l'agriculture promouvait l'agro-business, un comité de réflexion interministériel sur le foncier rural, ancré dans le ministère de l'agriculture, avait été mis en place. Ces deux espaces ont permis la construction progressive d'un réseau élargi d'acteurs, au sein et en dehors de l'administration, autour de la reconnaissance de la spécificité du foncier rural. L'un de ces experts, juriste de réputation internationale, bien connecté à la fois au monde rural et au monde politique, a convaincu le ministre de l'urgence de traiter la question. Celui-ci, numéro deux du gouvernement, a donné une forte légitimité au projet de réforme, que ses promoteurs ont voulu fonder sur un consensus le plus large possible, à partir de la reconnaissance de la diversité des groupes d'intérêts. Le « réseau sur enjeu » de départ est devenu une communauté de politique publique.

Les processus de réforme sont ainsi portés par des réseaux de politique publique, réunissant un ensemble plus ou moins large et cohérent d'acteurs. Ils peuvent s'accorder sur un référentiel ou s'opposer sur les visions politiques du secteur et ses enjeux. Ces réseaux réunissent chacun un ensemble évolutif d'acteurs et d'organisations, à travers des jeux de cooptation et d'intéressement, et traverse les frontières entre échelle nationale et internationale, administration et acteurs privés, experts et décideurs : une part importante des élites nationales est convertie au modèle libéral; techniciens des agences d'aide et des administrations nationales partagent fréquemment les mêmes cultures professionnelles et les mêmes corpus de référence, issus des prescriptions internationales et alimentés par les expériences nationales à travers études, évaluations, etc. Ces réseaux ont une histoire spécifique qui peut s'étendre sur plusieurs décennies et qui structure la nature des controverses, mais aussi la structure des arènes de débat sur les politiques. 
S'intéresser aux réseaux permet ainsi d'échapper au risque de réifier tant l'Etat et l'administration (en mettant en lumière les luttes interinstitutionnelles, les clivages politiques internes aux administrations sectorielles, et les liens, complexes, entre conflits de visions politiques et conflits institutionnels) que les institutions d'aide (au risque de sous-estimer les rivalités qui les opposent et de surestimer la cohérence idéologique de leurs actions) : au Bénin et au Burkina Faso, ce sont quasiment les mêmes institutions d'aide qui sont impliquées dans le secteur du foncier : 1'AFD, la GTZ, le $\mathrm{MCC}^{8}$. Mais leurs positionnements et leurs actions y ont été différents, pour des raisons qui tiennent à la fois au contexte national, à leur histoire dans le pays et aux personnes. Ainsi, le MCC lui-même a joué un rôle très différent dans les deux pays, soutenant le réseau de la privatisation et la formulation de la réforme foncière au Bénin dans un contexte de controverse sur le sens des politiques et d'absence de débat public, et au contraire finançant au Burkina Faso la mise en œuvre de la politique adaptative, en aval des choix politiques.

\section{Conclusion}

Toutes internationalisées et multi-acteurs qu'elles soient, il existe des politiques publiques dans les pays sous régime d'aide. Et l'analyse empirique de ces processus qui permet de mettre au jour la nature et les configurations des réseaux, le contenu des controverses et leurs enjeux, les rapports entre intérêts, idées et institutions, le poids normatif et financier de l'aide, le (souvent faible) rôle des mobilisations sociales dans la mises sur agenda et la définition des cadrages. Comparer les trajectoires de réformes de politiques publiques récentes, dans deux secteurs et deux pays voisins, questionne les interprétations trop mécanistes ou généralisatrices sur l'influence des bailleurs de fonds dans les pays « sous régime d'aide » ou sur la prégnance de la politique du ventre au sein d'Etats faiblement institutionnalisés. En Afrique pas plus qu'ailleurs (Crespy, Ravinet, 2014), les politiques publiques ne sont pas le strict reflet de ces intérêts, et le policy transfer n'est pas mécanique.

La formulation des réformes est ancrée dans la double histoire politique et institutionnelle des pays et du secteur d'action publique, le choix des modalités de mise en œuvre, et parfois les orientations même des réformes, résultent d'un processus complexe de "fabrique politique des politiques publiques » (Zittoun, 2013), produit de controverses, de conflits, parfois de mobilisations sociales, au sein de réseaux internationalisés. Ces controverses et ces conflits ne peuvent être réduits à des luttes d'intérêts de classe, corporatistes ou institutionnelles : elles recouvrent aussi, pour partie, des débats de polity.

Dans un même pays, cette fabrique politique des politiques publiques prend des formes différentes selon les secteurs. Ceci n'est pas surprenant en soi mais, faute d'analyses comparées dans un même pays, la mesure en est rarement prise dans les analyses sur l'Etat et les politiques publiques en Afrique. Surtout, leur spécificité ne tient pas seulement à la hiérarchie des professions en leur sein et à leur histoire institutionnelle : si les processus de réforme connaissent des trajectoires si différentes, entre reprise rapide des prescriptions internationales avec des nuances dans les dispositifs de mise en œuvre d'un côté, longues phases de maturations, violentes controverses et options divergentes de l'autre, c'est que la nature des enjeux politiques, au double sens des politics et de la polity, y sont très différents. En particulier, les Etats africains connaissent une pluralité des normes (Chauveau, Le Pape, Olivier de Sardan, 2001) structurelle et particulièrement marquée. Les différents secteurs d'action publique portent des enjeux spécifiques en termes de rapports entre Etat et citoyens,

\footnotetext{
${ }^{8}$ La Banque mondiale intervient au Burkina Faso à travers le Programme national de gestion des terroirs. Elle était parmi les financeurs du premier projet pilote au Bénin.
} 
de tensions entre régulations coutumières, étatiques et marchandes. Promouvant à des degrés divers l'institutionnalisation de régulations marchandes, les réformes des politiques publiques dans les années 2000 se confrontent à ces tensions, les exacerbent parfois, de façon particulièrement vive sur le secteur du foncier. Au delà des logiques corporatistes, des luttes interinstitutionnelles, des intérêts économiques des élites investissant dans la terre, c'est aussi un débat de société qui est sous-jacent aux luttes entre réseaux de politique publique, aux controverses et aux conflits qui les jalonnent, aux trajectoires chaotiques des tentatives de réforme, et qui expliquent que, sur ce champ, les prescriptions internationales (elles-mêmes hétérogènes) se concrétisent peu, ou très lentement.

La comparaison de deux trajectoires de réforme, sur un secteur aux enjeux forts en termes de politics et de polity, dans deux pays voisins confrontés globalement aux mêmes enjeux et avec l'intervention des mêmes acteurs internationaux amène à reconnaître la singularité des trajectoires de politiques, à questionner le postulat de la dépolitisation liée à l'aide (Ferguson, 1990), et celui de la soumission aux injonctions des bailleurs de fonds.

Notre analyse comparée éclaire en effet aussi la question des rapports entre bailleurs de fonds et institutions nationales. Certes, lorsque la survie des élites au pouvoir est liée à la rente de l'aide, " the governments [of weak states] have opted to accept their subordinate position and the inevitability of intimate donor involvment in policy making, and then pursue strategies to maximize their policy control within that context" (Whitfield, 2009b, p.356-357), ce qui les amène le plus souvent à éviter l'affrontement avec les bailleurs de fonds, à accepter - au moins en apparence - leurs injonctions aux réformes et leurs cadrages, quitte à privilégier les réinterprétations et inerties au moment de la mise en oeuvre. Mais une telle analyse néglige la spécificité des secteurs et leurs enjeux politiques: sur des sujets fortement politiques et controversés comme le foncier, les Etats ont à la fois des intérêts plus forts et une plus forte capacité à garder la main et à mettre à distance les injonctions, d'autant plus lorsque les positionnements des bailleurs de fonds sont contrastés et que les administrations peuvent jouer de ces contradictions. Dans de telles situations, les controverses ne portent pas que sur les modalités de mise en œuvre, mais sur l'orientation même des politiques. Lorsque le secteur est transversal à plusieurs administrations ou que son leadership est disputé, elles se doublent de luttes interinstitutionnelles, parfois feutrées parfois féroces, pour le contrôle de la politique et de ses orientations, et pour le contrôle des flux d'aide. En fonction des enjeux nationaux, mais aussi de l'histoire des controverses et de la dynamique des réseaux de politique publique, les trajectoires des politiques peuvent varier fortement d'un pays à l'autre, même lorsque les mêmes institutions d'aide sont impliquées.

S'intéresser à l'histoire du secteur, aux controverses qui le traversent au niveau national comme au niveau transnational, à la façon dont sont formulés les enjeux à ces deux échelles est ainsi nécessaire pour rendre compte de processus de production des politiques publiques, à la fois plus contingents et parfois moins «donor driven » qu'il n'apparaît à première vue, et pour mieux comprendre les modalités spécifiques que prennent «[the] expanded donor participation and the increasing entanglement of donors institutions and recipient administrative systems » (Whitfield, Fraser, 2009, p.19) caractéristiques des années 2000.

Enfin, interroger les processus de formulation des politiques publiques en Afrique confirme que les catégories clés de la sociologie politique de l'action publique (mise sur agenda, réseaux de politiques publiques, référentiel, parfois mobilisés ici de façon illustrative) sont à la fois pertinentes et éclairantes pour comprendre les processus complexes de coproduction de l'action publique et l'Etat en action en Afrique. A condition bien sûr de mener des enquêtes empiriques approfondies, prenant au sérieux les contextes historiques et politiques, l'enchâssement social et politique de l'Etat, les configurations internationalisées d'acteurs et les impacts de la présence structurelle de l'aide: la trajectoire des Etats africains, leurs 
modalités d'insertion dans les relations internationales, le degré d'institutionnalisation et d'autonomisation des administrations, les formes de mobilisation et de contestation diffèrent fortement des pays industrialisés. Désexotisant l'Afrique en lisant le «développement» comme une forme spécifique et internationalisée d'action publique (Lavigne Delville, 2016), les recherches sur les politiques publiques en Afrique peuvent en retour contribuer à déprovincialiser l'analyse des politiques publiques, en mettant ses concepts et ses théories à l'épreuve de configurations politiques nouvelles.

\section{Bibliographie}

ABRAHAMSEN R. (2004), "The World Bank's Good Governance Agenda. Narratives of Democracy and Power », in Gould J. et MARCusSEN H. S., ed., Ethnographies of Aid - Exploring Development Texts and Encounters, Roskilde, Roskilde University, p. 1544.

BANEgas R., 2003, La démocratie à pas de caméléon. Transitions et imaginaires politiques au Bénin, Paris, Karthala/CERI.

BARON C. et BonNASSIEUX A. (2013), Gouvernance hybride, participation et accès à l'eau potable Le cas des associations d'usagers de l'eau (AUE) au Burkina Faso, Annales de géographie, Armand Colin, p. 525-548.

BARON C. et MAILLEFERT M. (2011), «Une lecture institutionnaliste de la gouvernance de l'eau potable: des terrains d'Afrique de l'Ouest francophone aux faits stylisés », Regions \& Cohesion, 1 (3), p. 7-33.

BARON C. et TIDJANi Alou M. (2011), «L'accès à l'eau en Afrique subsaharienne: au-delà des modèles, une pluralité d'innovations locales », Mondes en développement (3), p. 7-22.

BAYART J.-F. (1999), « L'Afrique dans le monde: une histoire d'extraversion », Critique internationale, 5 (1), p. 97-120.

Bergamaschi I. (2009), "Mali : Patterns and Limits of Donor-driven Ownership », in Whitfield L., ed., The Politics of Aid. African Strategies for Dealing with Donors, Oxford, Oxford University Press, p. 217-245.

BIERSCHENK T. (2014), "From the anthropology of development to the anthropology of global social engineering », Zeitschrift für Ethnologie, 139 (1), p. 73-97.

Bierschenk T. et Olivier De SARdAn J.-P. (ed.) (2014), States at Work. Dynamics of African Bureaucracies, Vol. 12, Leiden, Brill.

BonnassieuX A. et GANGNERON F. (2011), « Des mini-réseaux d'eau potable : entre enjeux politiques et arrangements locaux. Le cas de la commune de Djougou au Bénin », Mondes en développement, 155 (3), p. 77-92.

Boone C., 2013, Property and Political Order in Africa. Land Rights and the Structure of Politics, Cambridge, Cambridge University Press.

BRUCE J. W. et FREUDENBERGER M. S. (1992), Institutional opportunities and constraints in African land tenure: shifting from a 'replacement' to an 'adaptation' paradigm, Land Tenure Center, University of Wisconsin, Madison.

BuiJtenhuijs R. et THIRIOT C. (1995), Démocratisation en Afrique au sud du Sahara 19921995 (un bilan de la littérature), Talence/Leiden CEAN/Afrika Studiecentrum, 217 p.

Chauveau J.-P., Le PAPE M. et Olivier De SARdan J.-P. (2001), « La pluralité des normes et leurs dynamiques en Afrique », in WINTER G., ed., Inégalités et politiques publiques en Afrique. Pluralité de normes et jeux d'acteurs, Paris, Karthala, p. 145-162. 
Cling J.-P., RaZafindrakoto M. et Roubaud F. (2002), « La Banque Mondiale et la lutte contre la pauvreté : "tout changer pour que tout reste pareil ?" », Politique africaine, 87.

Coussy J. (2006), «Etats africains, programmes d'ajustement et consensus de Washington », L'Economie Politique (4), p. 29-40.

CRESPY A. et RAVINET P. (2014), « Les avatars du néo-libéralisme dans la fabrique des politiques européennes », Gouvernement et action publique, 2 (2), p. 9-29.

DARbon D. (ed.) (2009), La politique des modèles en Afrique. Simulation, dépolitisation et appropriation, Paris/Pessac, Karthala/MSHA.

DARBON D. (2004), "Peut-on relire le politique en Afriques via les politiques publiques? ou « Policies make politics »: Does it make sense in African Countries ?", in Triulzi A. et Ercolessi C., ed., State, power, and new political actors in postcolonial Africa, Milano, Fondazione Giangiacomo Feltrinelli, p. 175-199.

DARBON D. (2015), L'extranéité des politiques comme ressource de la formation de l'Etat et de la société projetés, Working paper, Toujouse, ANR APPI.

DARBon D. et Crouzel I. (2009), « Administrations publiques et politiques publiques des Afriques », in GAZIBO M. et THIRIOT C., ed., Le politique en Afrique, Paris, Karthala, p. 71-102.

Diop M. et Hamath DiA A. (2011), «Réformes des services d'eau en milieu rural africain : enjeux et limites du montage institutionnel de gestion. Une étude de cas au Sénégal », Mondes en développement, 155 (3), p. 37-58.

Евоко F. (2015), Repenser l'action publique en Afrique, Paris, Karthala.

ENGUELEGUELE M. (2008), « Quelques apports de l'analyse de l'action publique à l'étude du politique en Afrique subsaharienne », Politique et sociétés, 27 (1), p. 3-28.

FERguson J. (1990), The Anti-Politics Machine: 'Development', Depoliticization and Bureaucratic Power in Lesotho, Minneapolis, University of Minnesota Press.

FouILlEuX E. (2015), « Au delà des Etats en action... La fabrique des politiques publiques globales », in BoussagueT L., JACQUOT S. et RAVINET P., ed., Une" French touch" dans l'analyse des politiques publiques?, Paris, Presses de Sciences Po, p. 287-318.

Fouilleux E. et Jobert B. (2017), « Pour une approche agonistique des débats de politique publique. Le cheminement des controverses dans la globalisation néolibérale », Gouvernement et action publique, 6 (3), p. 9-36.

Hibou B. (1998), « Retrait ou redéploiement de l'Etat? », Critique internationale, 1998/4 (1), p. 151-168.

Hilgers M. et Mazzocchetti J. (ed.) (2010), Révoltes et oppositions dans un régime semiautoritaire: le cas du Burkina Faso, Paris, Karthala.

Hоснет P. et JАСОВ J.-P. (2014), «Quelle place pour la reconnaissance des droits coutumiers dans la réforme foncière burkinabé ? Entre substitution et adaptation », Colloque final du projet de recherche ANR APPI, Toulouse, décembre 2014.

Igue O. J. et Soule B. G. (1992), L'Etat entrepôt au Bénin: commerce informel ou solution à la crise?, Paris, Karthala. 
JАСОВ J.-P. (2013), "Suis-je le gardien de mon frère? ». L'émergence de la relation sujetobjet dans la législation foncière burkinabè de 2009, Les Cahiers du Pôle Foncier $\mathrm{n}^{\circ}$ 4, Montpellier, Pôle foncier de Montpellier, 20 p.

JAMPY V. (2012), Le gouvernement expert de l'aide publique au développement : pratiques et représentations des "développeurs" au Sénégal, Doctorat de Science Politique Université Paris I - Panthéon Sorbonne, Paris, 511 p.

JOBERT B. (1998), «La régulation politique : le point de vue d'un politiste », in COMMAILLE J. et JOBERT B., ed., Les métamorphoses de la régulation politique, Paris, LGDJ, p. 119143.

LAVigne Delville P. (2010), «La réforme foncière rurale au Bénin. Émergence et mise en question d'une politique instituante dans un pays sous régime d'aide », Revue française de science politique, 60 (3), p. 467-491.

Lavigne Delville P. (2016), « Pour une socio-anthropologie de l'action publique dans les pays 'sous régime d'aide' », Anthropologie \& développement (45), p. 33-64.

Lavigne Delville P. et AbDelKader A. (2010), "A cheval donné, on ne regarde pas les dents ». Les mécanismes et les impacts de l'aide vus par des acteurs nigériens, Etudes et Travaux $\mathrm{n}^{\circ} 83$, Niamey, LASDEL, $113 \mathrm{p}$.

Lavigne Delville P. et SaÏAh C. (2016), Politiques foncières et mobilisations sociales au Bénin. Des organisations de la société civile face au Code domanial et foncier, Cahier $\mathrm{du}$ Pôle foncier $\mathrm{n}^{\circ}$ 14, Montpellier, Pole Foncier, $60 \mathrm{p}$.

Lavigne Delville P. et Thieba D. (2015), « Débat public et production des politiques publiques au Burkina Faso. La Politique nationale de sécurisation foncière », Participations, 11 (1), p. 213-236.

LE Roy E. (1985), « La loi sur le domaine national a vingt ans: joyeux anniversaire ? », Mondes en développement, 13 (52), p. 667-685.

LECA J. (2012), « L'état entre politics, policies et polity. ou peut-on sortir du triangle des Bermudes ?», Gouvernement et action publique, 1 (1), p. 59-82.

MANJI A. (2006), The politics of land reform in Africa: from communal tenure to free markets, London/New York, Zed Books.

MAthieu P. (1996), «Pratiques informelles, gestion de la confusion et invention du foncier en Afrique », in DE VILLERS G., ed., Phénomènes informels et dynamiques culturelles en Afrique, Paris, Cedaf-L'Harmattan, p. 64-87.

MEDARD J.-F. (1991a), « L'État néo-patrimonial en Afrique noire », in MEDARD J.-F., ed., États d'Afrique noire. Formations, mécanismes et crise, Paris, Karthala, p. 323-353.

Muller P. (2010), « Référentiel », in Boussaguet L., JACQuot S. et RAVINET P., ed., Dictionnaire des politiques publiques, Paris, Presses de Sciences Po, p. 555-562.

Muller P. (2010), "Secteur », in Boussaguet L., JacQuot S. et Ravinet P., ed., Dictionnaire des politiques publiques, Paris, Presses de Sciences Po, p. 591-599.

OliviER DE SARDAN J.-P. (1995), Anthropologie et développement. Essai en anthropologie du changemement social, Paris, APAD/Karthala.

Olivier de SARDAN J.-P. et DAgOBi E. H. A. (2000), « La gestion communautaire sert-elle l'intérêt public? Le cas de l'hydraulique villageoise au Niger ", Politique africaine (80), p. 153-168. 
POMMEROLLE M.-E. (2010), «The extraversion of protest: conditions, history and use of the 'international' in Africa », Review of African political economy, 37 (125), p. 263-279.

Provini O. et SCHLIMMER S. (2016), « Négocier l'action publique dans un État sous régime d'aide: une analyse comparée des politiques de l'enseignement supérieur et du foncier en Tanzanie », Revue internationale de politique comparée, 23 (2), p. 199-223.

SHIPTON P. et GOHEEN M. (1992), «Introduction. Understanding African Land-Holding: Power, Wealth, and Meaning », Africa: Journal of the International African Institute, 62 (3), p. 307-325.

TAllet B. (2009), « La Réorganisation Agraire et Foncière au Burkina Faso (1984-2004). Vingt ans de décalage entre cadre légal et pratiques locales », in COLIN J.-P., LE MeUR P.-Y. et LEONARD E., ed., Les politiques d'enregistrement des droits fonciers. Du cadre légal aux pratiques locales, Paris, Karthala, p. 141-166.

Thatcher M. (2004), " Réseau (policy network) », ed., Dictionnaire des politiques publiques, Presses de Sciences Po (PFNSP), p. 384-390.

Thomas M. A. (2004), « Can the World Bank Enforce its Own Conditions? », Development \& Change, 35 (3), p. 485-497.

VAlette H., BARON C., EnTEN F., et al (ed.) (2015), Une action publique éclatée ? Production et institutionnalisation de l'action publique dans les secteurs de l'eau potable et du foncier - Burkina Faso, Niger, Bénin, Nogent sur Marne/Toulouse, GRET/LEREPS.

Valette H., Gangneron F. et Bonnassieux A. (2016), « L'intégration et la mise en œuvre des principes marchands dans le secteur de l'eau en milieu rural et semi-urbain béninois. Une analyse des réformes et des pratiques », Anthropologie \& développement (45), p. 113-142.

Whitfield L. (ed.) (2009a), The Politics of Aid. African Strategies for Dealing with Donors, Oxford/New York, Oxford University Press.

WhitField L. (2009b), « Conclusion: Changing Conditions? », in WhitFIELD L., ed., The Politics of Aid, Oxford, Oxford University Press, p. 361-379.

Whitfield L. et Fraser A. (2009), «Introduction: Aid and Sovereignty », in Whitfield L., ed., The Politics of Aid, Oxford, Oxford University Press, p. 1-26.

ZitToun P. (2013), La fabrique politique des politiques publiques, Paris, Presses de Sciences Po. 